\title{
THE ASYMPTOTIC SOLUTIONS OF A LINEAR DIF- FERENTIAL EQUATION OF THE SECOND ORDER WITH TWO TURNING POINTS $\left({ }^{1}\right)$
}

\author{
BY \\ RUDOLPH E. LANGER
}

1. Introduction. This paper is concerned with the functional forms, namely with the leading terms of asymptotic representations, of the solutions of a differential equation

$$
\frac{d^{2} y}{d s^{2}}+\left\{\lambda^{2} \theta^{2}(s)+\mathrm{X}(s, \lambda)\right\} y=0
$$

in which

(i) $\lambda^{2}$ is a large complex parameter;

(ii) $s$ is the real variable over some interval $a \leqq s \leqq b$;

(iii) $\theta^{2}(s)$ is a real function which has precisely two zeros, both simple, on the interval $(a, b)$;

(iv) $\mathrm{X}(s, \lambda)$ is bounded in $s$ and $\lambda$, and is integrable in $s$.

The zeros of $\theta^{2}(s)$ are referred to as turning points of the differential equation.

The solution forms of a differential Equation (1.1) over an interval that includes just one turning point are known, and can be expressed asymptotically with respect to $\lambda$ through the use of Bessel functions. That being so, it could well seem that forms having validity over the entire interval with two turning points might be obtainable by dealing with the interval by halves, and, in the familiar way, patching together the forms appropriate to the respective halves, along with their derivatives. That procedure, however, is not practicable when the forms in hand are not actual representations but only the leading terms of asymptotic ones. For such a form is explicit only to a certain degree, and because of that ordinarily represents not just a single particular solution over an appropriate $x$-interval, but a whole infinity of them. In other sub-intervals these solutions may be wholly dissimilar. To fit together two such forms, along with their derivatives, at some chosen point between the two turning points, therefore does not identify the solutions to which that fit applies, and accordingly cannot be taken as a basis for inferring the continuations of those solutions in intervals beyond the turning points.

In the present paper the interval is not treated by halves. It is shown that forms which are valid over the whole interval can be given in terms of ap-

Received by the editors April 15, 1957.

(1) Sponsored by the United States Army under Contract No. DA-11-022-ORD-2059. 
propriate Weber functions $w(z, \kappa)$, namely of appropriate solutions of Weber's differential equation

$$
\frac{d^{2} w}{d z^{2}}+\left\{2 \kappa+1-z^{2}\right\} w=0 .
$$

These Weber functions are thus shown to fill, in the instance of two turning points, a role that is analogous to that which the Bessel functions fill when there is only one.

A study of the solution forms of the Weber Equation (1.2) with both the variable and the parameter complex, and the latter large, was made by Schwid, [1] $\left({ }^{2}\right)$, and more recently by Erdélyi, Kennedy and McGregor, [2]. References to the literature are given by these authors, who also remark that differential equations of the type (1.1) present themselves in quantum mechanics, and in problems of wave motion, of diffraction, of vibrations, etc. No use of these other studies is, however, made in the present paper. The reasons are that the solutions singled out and set forth in them are not optimal ones for the present investigation, and that the solution forms given by them, applying as they do to domains of the complex variable, are of greater intricacy than is here needed. The particular Weber functions that assume a basic role here, are ones that have monotonic absolute values, either on each side of a turning point, or over the given interval as a whole.

The cases in which the parameter $\lambda^{2}$ is real are special ones. There are two of them, and these are quite distinct. If $\lambda^{2} \theta^{2}(s)$ is negative between the turning points, the solutions of the equation are of an exponential character there, and make transitions to oscillatory forms when the turning points are traversed. An earlier study of the Equation (1.1) in this case was made, under the author's direction, by Johnson, [3].

When $\lambda^{2} \theta^{2}(s)$ is positive between the turning points the solutions oscillate there, and are continued by exponential forms beyond the turning points. In this case a certain exceptional phenomenon occurs. The solutions of the differential equation are functions of $s$ and $\lambda$. Since the interval for the variable $s$ is closed and includes no singular point of the differential equation, each solution, with $\lambda$ fixed, is bounded. However, in general, for complex as well as for real $\lambda$, the solutions $y(s, \lambda)$ when normalized to take on a prescribed value independent of $\lambda$ at some specified point $s$, are all unbounded as to $\lambda$, namely are not uniformly bounded as to $s$. The exception which occurs, when $\lambda^{2} \theta^{2}(s)$ is positive between the turning points, is that a certain infinite set of characteristic $\lambda$-values then presents itself with the property that the associated solution set is uniformly bounded. The phenomenon is well known in the case of the special Equation (1.2). For in the instance of that, the positive integral values of $\kappa$ are characteristic, in that the equation then admits as respective solutions the bounded functions $e^{-z^{2} / 2} P_{k}(z)$, in which the factors

(2) Numerals within brackets will be used to refer to the bibliography attached. 
$P_{\kappa}(z)$ are polynomials - the Hermite polynomials of the degree $\kappa$.

The method which we apply to the Equation (1.1) is based upon a comparison of this equation-in a suitably normalized form-with a so-called related equation. This latter is a transformed Weber equation. On the ground of that comparison we deduce the solution forms of the one equation from the corresponding known ones of the other.

The hypotheses upon the differential Equation (1.1) under which the investigation is to be pursued are the following:

(a) Over a given interval $a \leqq s \leqq b$, the function $\theta^{2}(s)$ is of the form

$$
\theta^{2}(s)=(s-\alpha)(s-\beta) \theta_{1}^{2}(s),
$$

wherein

$$
a<\alpha<\beta<b,
$$

while the factor $\theta_{1}^{2}(s)$ is a real function that is bounded from zero, and is possessed of a continuous third derivative, namely, is of the class $C^{\prime \prime \prime}$.

(b) The parameter $\lambda^{2}$ exceeds some appropriately large constant in absolute value, but is otherwise eligible to take on all complex values.

(c) The function $\mathrm{X}(s, \lambda)$ is bounded as to $s$ and $\lambda$, and is integrable in $s$.

We shall derive the forms of the solutions of the differential equation (1.1) to a degree of explicitness that extends only to the leading (dominant) terms of their asymptotic representations. Therefore the dependence upon $\lambda$ that is introduced into the Equation (1.1) by the coefficient $\mathrm{X}(s, \lambda)$ will remain below the threshold of explicitness, and will not assume quantitative significance in the discussion. The elements $\theta(s)$ and $\lambda$ therefore remain only partially defined. For the differential equation specifies them merely as appropriate factors of the product $\lambda^{2} \theta^{2}(s)$, and that leaves undetermined the choices of square roots and the distribution of constant factors. To make $\theta(s)$ and $\lambda$ completely specific, we therefore adopt the normalizing relation

$$
\int_{\alpha}^{\beta} \theta(s) d s=\frac{\pi i}{2}
$$

and the determinations

$$
\arg \theta(s)=\left\{\begin{array}{cl}
0, & \text { for } s<\alpha, \\
\pi / 2, & \text { for } \alpha<s<\beta \\
0, & \text { for } \beta<s
\end{array}\right.
$$

and

$$
0 \leqq \arg \lambda<\pi .
$$

Consistently with these we shall take 


$$
\arg \left\{\int_{\beta}^{s} \theta(s) d s\right\}=\left\{\begin{array}{cl}
0, & \text { for } \beta<s, \\
3 \pi / 2, & \text { for } \alpha<s<\beta .
\end{array}\right.
$$

2. The functions $x(s)$ and $s(x)$. Let $x$ be a real variable, and the functions $\phi$ and $\Phi$ be defined thus

$$
\begin{aligned}
& \phi(x)=\left(x^{2}-1\right)^{1 / 2} \\
& \Phi(x)=\int_{1}^{x} \phi(x) d x
\end{aligned}
$$

with the determinations

$$
\begin{aligned}
& \arg \phi(x)=\left\{\begin{array}{cl}
0, & \text { for } \quad x<-1, \\
\pi / 2, & \text { for }-1<x<1, \\
0, & \text { for } 1<x,
\end{array}\right. \\
& \arg \Phi(x)=\left\{\begin{array}{cl}
0, & \text { for } 1<x, \\
3 \pi / 2, & \text { for }-1<x<1 .
\end{array}\right.
\end{aligned}
$$

Then consider the equation

$$
\int_{1}^{x}\left(x^{2}-1\right)^{1 / 2} d x=\int_{\beta}^{s} \theta(s) d s .
$$

It establishes a correspondence of $x=1$ with $s=\beta$ and as $x$ and $s$ increase from these initial values its members likewise increase, continuously and monotonically. It therefore maps the sub-interval $\beta \leqq s \leqq b$, upon a certain interval $1 \leqq x \leqq x_{b}$. As $x$ and $s$ decrease from the values 1 and $\beta$ the members of the equation assume pure imaginary values with the fixed argument $3 \pi / 2$, while their moduli increase continuously. They attain the values $-\pi i / 2$ when $x=-1$ and $s=\alpha$, and thus the equation maps the sub-interval $\alpha \leqq s \leqq \beta$ upon the interval $-1 \leqq x \leqq 1$. By virtue of this, the equation is seen to be alternatively expressible in the form

$$
\int_{-1}^{x}\left(x^{2}-1\right)^{1 / 2} d x=\int_{\alpha}^{s} \theta(s) d s .
$$

From this it is readily seen to map the sub-interval $a \leqq s \leqq \alpha$ upon a certain interval $-x_{a} \leqq x \leqq-1$.

The implicit relation (2.3) thus defines $x(s)$ and $s(x)$ as continuous increasing functions on the respective intervals $a \leqq s \leqq b$ and $-x_{a} \leqq x \leqq x_{b}$. Except at the turning points $s=\alpha, s=\beta$, and their correspondents $x=-1, x=1$, the differentiability of these functions, the fact that they are of the class $C^{\prime \prime \prime}$, and the formulas

$$
x^{\prime}(s)=\theta(s) / \phi(x), \quad s^{\prime}(x)=\phi(x) / \theta(s),
$$


may be inferred by elementary considerations. We shall show, in the following section, that the facts are the same at the points which, in the remarks just made, were omitted as conceivably exceptional.

3. The function $x(s)$ at the turning points. Let $s$ be taken in any deleted neighborhood of $s=\beta$ in which it is bounded from the point $s=\alpha$. In this neighborhood $\theta(s)$ has the form

$$
\theta(s)=(s-\beta)^{1 / 2} \omega(s),
$$

with a $\omega(s)$ a positive function, which, under the hypothesis (a) of $\$ 1$ is of the class $C^{\prime \prime \prime}$. The function $\Omega(s)$ defined by the formula

$$
\Omega(s)=\left(\int_{\beta}^{s} \theta(s) d s\right) /(s-\beta)^{3 / 2},
$$

is positive in this neighborhood. It is made to be continuous $\left({ }^{3}\right)$ at $s=\beta$ by the value assignment

$$
\Omega(\beta)=2 \omega(\beta) / 3 .
$$

The differentiation of the formula (3.1) at $s \neq \beta$, followed by an integration by parts, yields the formula

$$
\Omega^{\prime}(s)=\left(\int_{\beta}^{s}(s-\beta)^{3 / 2} \omega^{\prime}(s) d s\right) /(s-\beta)^{5 / 2},
$$

whereas, by (3.1) and (3.2),

$$
\frac{\Omega(s)-\Omega(\beta)}{s-\beta}=\frac{\int_{\beta}^{s}(s-\beta)^{1 / 2}\{\omega(s)-\omega(\beta)\} d s}{(s-\beta)^{5 / 2}} .
$$

From this latter

$$
\Omega^{\prime}(\beta)=\frac{2}{5} \omega^{\prime}(\beta),
$$

and since this is the limiting form of (3.3), we see that $\Omega(s)$ is at least of the class $C^{\prime}$. The procedure we have thus applied to $\Omega(s)$ is repeatable, first upon $\Omega^{\prime}(s)$ and then, in turn, upon $\Omega^{\prime \prime}(s)$. It is thus found that $\Omega(s)$ is of the class $C^{\prime \prime \prime}$.

The function $\Phi(x)$, as is shown by the formulas (2.1), is of the form

$$
\Phi(x)=(x-1)^{3 / 2} \Phi_{1}(x),
$$

with $\Phi_{1}(x)$ positive and indefinitely differentiable in any neighborhood of the

(3) The limiting values of the indeterminate forms in this section are all obtainable by the elementary method of replacing the numerator and denominator by their derivatives before proceeding to the limit. 
point $x=1$ in which $x$ is bounded from $x=-1$. By virtue of this and the evaluation (3.1), the form of the relation (2.3) is seen to be

$$
(x-1)^{3 / 2} \Phi_{1}(x)=(s-\beta)^{3 / 2} \Omega(s) .
$$

Now $\Phi_{1}(x)$ and $\Omega(s)$, since they are positive and of the class $C^{\prime \prime \prime}$ have each a cube root that is real and of the same class. In terms of these the relation may be expressed as

$$
(x-1) \Phi_{1}^{2 / 3}(x)=(s-\beta) \Omega^{2 / 3}(s),
$$

and to this form the elementary implicit function theorem is applicable even at $s=\beta, x=1$. The formula for $x^{\prime}(s)$ that is thus obtainable, is twice differentiable, and gives at $s=\beta$ the value

$$
x^{\prime}(\beta)=\left\{\Omega(\beta) / \Phi_{1}(1)\right\}^{2 / 3},
$$

which is positive.

The considerations we have thus applied to neighborhoods of the point $s=\beta$ are easily rephrased to apply to neighborhoods of $s=\alpha$. It is convenient in that case to take the relation between $x$ and $s$ in the form (2.3a). The functions $x(s)$ and $s(x)$ thus have nonvanishing first derivatives, and are of the class $C^{\prime \prime \prime}$ over the entire respective intervals of $s$ and $x$.

4. A normalization of the given differential equation. The related equation. The change of variables

$$
s=s(x), \quad y(s)=\left\{s^{\prime}(x)\right\}^{1 / 2} u(x),
$$

transforms the differential equation (1.1) into

$$
\frac{d^{2} u}{d x^{2}}+\left\{\lambda^{2} \theta^{2}(s) s^{\prime 2}+\mathrm{X}(s, \lambda) s^{\prime 2}+\frac{s^{\prime \prime \prime}}{2 s^{\prime}}-\frac{3}{4}\left(\frac{s^{\prime \prime}}{s^{\prime}}\right)^{2}\right\} u=0 .
$$

Because of the relation (2.4) this is, in fact,

$$
u^{\prime \prime}+\left\{\lambda^{2} \phi^{2}(x)+g(x, \lambda)\right\} u=0,
$$

with

$$
g(x, \lambda)=\mathrm{X}(s(x), \lambda) s^{\prime 2}(x)+\frac{s^{\prime \prime \prime}(x)}{2 s^{\prime}(x)}-\frac{3}{4}\left(\frac{s^{\prime \prime}(x)}{s^{\prime}(x)}\right)^{2} .
$$

Under the hypothesis (c) of $\S 1$, and because $s^{\prime}(x)$ is bounded from zero and $s^{\prime \prime \prime}(x)$ is continuous, the function $g(x, \lambda)$ is integrable in $x$ over the interval $\left(-x_{a}, x_{b}\right)$, and is bounded when $|\lambda|$ is sufficiently large. We shall refer to (4.2) as the normal form of the given differential equation. It will be convenient to use this form as the basis of our analysis. In the end the return to the original form (1.1) will easily be accomplished.

Under the change of variables and parameter 


$$
z=(2 \kappa+1)^{1 / 2} x, \quad w(z)=v(x),
$$$$
2 \kappa+1=i \lambda,
$$

the Weber differential Equation (1.2) is transformed into

$$
v^{\prime \prime}+\lambda^{2} \phi^{2}(x) v=0 .
$$

Because of the resemblance of this to the normalized given Equation (4.2), we shall refer to it as the related differential equation.

5. Notations and remarks. On an interval whose closure contains only the one zero, $x=1$, of the coefficient $\phi^{2}(x)$, the differential Equation (4.4) fulfills certain conditions, [1], under which its solution forms are known and are readily brought to hand, [4]. These forms are conveniently expressed in terms of the functions

$$
\begin{aligned}
\Psi(x) & =\Phi^{1 / 6}(x) / \phi^{1 / 2}(x), \\
\xi(x) & =\lambda \Phi(x),
\end{aligned}
$$

and these functions we must therefore discuss. As to $\Psi(x)$, it is to be observed that its singularity at the zero of $\phi(x)$ is removable, since $\Phi(x)$ also vanishes there, and to the proper order. With the appropriate value assigned to it at $x=1$ this function is continuous, even differentiable, and is bounded from zero.

As to $\xi(x)$ the solution forms are various, depending in the first instance upon whether or not $|\xi| \leqq N$, where $N$ is a constant which we think of as large. The precise numerical value of $N$ will not be brought into question. When $|\xi|>N$, the forms depend further upon the domain of arg $\xi$ as will be specified below.

As a function of $x$ the modulus of $\xi$ is seen, from (5.1) and (2.1), to vanish at $x=1$, and to increase monotonically and indefinitely as $x$ recedes from $x=1$ in either the positive or the negative direction. There are, therefore, two points, with respective abscissas $x_{0}$ and $x_{1}$, of which $x_{0}<1$, and $x_{1}>1$, at which $|\xi(x)|=N$, and between which $|\xi(x)|<N$. Because $|\xi(x)|$ is of the order of $\left|\lambda(x-1)^{3 / 2}\right|$ near the point $x=1$, it is seen that the intervals $\left(x_{0}, 1\right)$ and $\left(1, x_{1}\right)$, and hence also $\left(x_{0}, x_{1}\right)$ are of lengths that are of the order of $N|\lambda|^{-2 / 3}$. Whatever the constant $N$ may be that is in question, the relations $0<x_{0}, x_{1}<x_{b}$, and $x_{1}<x_{a}$, maintain when $|\lambda|$ is sufficiently large. We shall require $\lambda$ to fulfill this condition. In the interval $\left(x_{0}, x_{1}\right)$ then, $|\xi(x)| \leqq N$, and in the interval $\left(-x_{1},-x_{0}\right)$ accordingly $|\xi(-x)| \leqq N$. In each of these intervals $\Psi(|x|)$ and $1 / \Psi(|x|)$ are bounded.

For values of $\xi$ whose modulus exceeds a suitably large $N$ the solutions of the Equation (4.4) are representable asymptotically by forms which depend upon the sector of a set $\Xi^{(k)}$ in which the point $\xi$ lies. These sectors are defined by the relations

$$
\Xi^{(k)}:(k-1) \pi+\epsilon \leqq \arg \lambda \leqq(k+1) \pi-\epsilon,
$$$$
k=0, \pm 1, \pm 2, \cdots,
$$

in which $\epsilon$ is a positive but arbitrarily small constant. It is clear that the 
sectors (5.2) overlap, and that therefore $\xi$ may well lie in two of them. The location of $\xi$, then, either identifies an index $k$ or conforms with two consecutive ones. When the latter is the case, either one of the indices in question may be chosen as applicable, and the choice is immaterial. For, so long as two indices apply, the forms given by them respectively are asymptotically equivalent.

The configuration of values $\xi$ which constitutes the map of the $x$-interval is easily determined. By (5.1) we have $\arg \xi=\arg \Phi+\arg \lambda$. By (2.2) and (2.1) the half-line $1<x$ corresponds to $0<\Phi$, and the interval $-1 \leqq x \leqq 1$ corresponds to the straight segment on which $\arg \Phi=3 \pi / 2,|\Phi| \leqq \pi / 2$. Finally, for $x<-1$ the difference $\Phi(x)-\Phi(-1)$ is negative and of increasing absolute value with decreasing $x$. The locus of $\xi$ in the complex plane is thus as shown in Fig. 1.

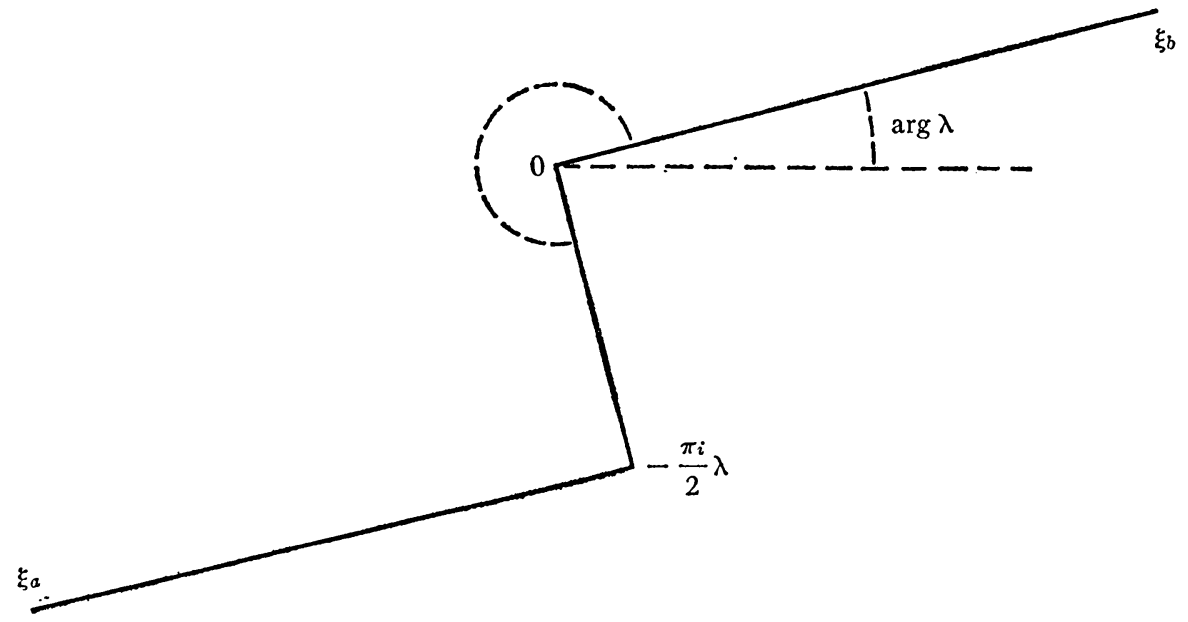

FIG. 1

The function $\lambda^{1 / 6} \phi^{1 / 2}(x)$ is, by (5.1), equal to $\xi^{1 / 6}(x) / \Psi(x)$. Since it is also even in $x$ it is seen that

$$
\frac{1}{\lambda^{1 / 6} \phi^{1 / 2}(x)}=\frac{\Psi(|x|)}{\xi^{1 / 6}(|x|)}
$$

The left-hand member of this equation, which will appear in some of the formulas to be given, is thus bounded on the intervals upon which $|\xi(x)|>N$ or $|\xi(-x)|>N$, despite the fact that on such intervals the bounds of $x$ from the zeros of $\phi(x)$ may diminish with $1 /|\lambda|$.

We shall use the symbol $B$ in the manner $B(\lambda), B(x, \lambda), B(x, t, \lambda)$ etc. to signify, in a generic sense, a (some) function which is bounded when $|\lambda|$ is 
large, and uniformly so with respect to such other variables as it may depend upon. Finally, we shall use the notation $[\sigma]$ as an abbreviation in the sense

$$
[\sigma]=\sigma+\frac{B(x, \lambda)}{\lambda}+\frac{B(x, \lambda)}{\xi} .
$$

6. On solutions of the related equation. Over an interval whose closure includes the turning point $x=1$ but not $x=-1$ the forms of the solutions $v(x)$ of the differential equation (4.4) are known, [4]. And likewise known are the forms of certain respective functions $\bar{v}(x)$, in terms of which the derivatives are given by the formula

$$
v^{\prime}(x)=\frac{i \lambda^{2 / 3}}{\Psi^{2}(x)} \bar{v}(x)+\frac{\Psi^{\prime}(x)}{\Psi(x)} v(x) .
$$

For our purposes it is essential to have at hand the forms of certain particular solutions, and to have these for the entire $x$-interval. We proceed to derive them. If the procedure seems circuitous, that is because of the particularity of the solutions that are to be singled out.

When $x$ is on the interval $\left(-x_{0}, x_{0}\right)$ it is to be observed from Fig. 1 that the point $\xi(x)$, which lies on the central segment of the broken line, is in the sector $\boldsymbol{\Xi}^{(2)}$ of (5.2). For such $\xi$ the Equation (4.4) has a solution $\left({ }^{4}\right)$, which we shall designate by $v_{-1}(x)$ that has the form

$$
\begin{aligned}
& v_{-1}(x)=\frac{1}{\lambda^{1 / 6} \phi^{1 / 2}(x)} e^{-i \xi(x)}[1], \\
& \bar{v}_{-1}(x)=\frac{-\lambda^{1 / 6} \Phi^{1 / 3}(x)}{\phi^{1 / 2}(x)} e^{-i \xi(x)}[1] .
\end{aligned}
$$

The relation (6.1) accordingly gives

$$
v_{-1}^{\prime}(x)=\frac{1}{\lambda^{1 / 6} \phi^{1 / 2}(x)}\left\{-i \lambda \phi(x)[1]+\frac{\Psi^{\prime}(x)}{\Psi(x)}[1]\right\} e^{-i \xi(x)},
$$

and this, with the use of the abbreviation (5.4), can be written as

$$
v_{-1}^{\prime}(x)=\frac{-i \lambda}{\lambda^{1 / 6} \phi^{1 / 2}(x)} e^{-i \xi(x)}[\phi(x)] .
$$

When $x$ is on the interval $1<x$, the point $\xi(x)$ lies on the right-hand segment of the broken line in Fig. 1, and is therefore in the sector $\Xi^{(0)}$ of (5.2) when $0 \leqq \arg \lambda \leqq \pi-\epsilon$, and in $\Xi^{(1)}$ when $\epsilon \leqq \arg \lambda<\pi$. In these sectors the form of $v_{-1}(x)$ is, $[4,(31)]$,

(4) This is the solution that is denoted by $u_{2,2}(z)$ in $[4,(21)]$. 


$$
\begin{aligned}
& v_{-1}(x)=\frac{1}{\lambda^{1 / 6} \phi^{1 / 2}(x)}\left\{e^{i \xi(x)}[\delta]+e^{-i \xi(x)}[1]\right\}, \\
& \bar{v}_{-1}(x)=\frac{\lambda^{1 / 6} \Phi^{1 / 3}(x)}{\phi^{1 / 2}(x)}\left\{e^{i \xi(x)}[\delta]-e^{-i \xi(x)}[1]\right\},
\end{aligned}
$$

wherein

$$
\delta=\left\{\begin{aligned}
-1, & \text { when } 0 \leqq \arg \lambda \leqq \pi-\epsilon, \\
0, & \text { when } \epsilon \leqq \arg \lambda<\pi .
\end{aligned}\right.
$$

From this and (6.1)

$$
v_{-1}^{\prime}(x)=\frac{i \lambda}{\lambda^{1 / 6} \phi^{1 / 2}(x)}\left\{e^{i \xi(x)}[\delta \phi(x)]-e^{-i \xi(x)}[\phi(x)]\right\} .
$$

In the intermediate interval $\left(x_{0}, x_{1}\right)$, namely in the neighborhood of the turning point $x=1$, the forms are, [4, p. 461],

$$
\begin{aligned}
& v_{-1}(x)=\Psi(x) \xi^{1 / 3}\left\{\gamma_{1,1} J_{-1 / 3}(\xi)+\gamma_{2,1} J_{1 / 3}(\xi)\right\}+\frac{B(x, \lambda)}{\lambda}, \\
& \bar{v}_{-1}(x)=i \Psi(x) \xi^{2 / 3}\left\{\gamma_{1,1} J_{2 / 3}(\xi)-\gamma_{2,1} J_{-2 / 3}(\xi)\right\}+\frac{B(x, \lambda)}{\lambda},
\end{aligned}
$$

with the appropriate coefficients from the set

$$
\begin{aligned}
\gamma_{1,1} & =\left(\frac{2 \pi}{3}\right)^{1 / 2} e^{-\pi i / 4}, & \gamma_{2,1} & =\left(\frac{2 \pi}{3}\right)^{1 / 2} e^{-\pi i / 4}, \\
\gamma_{1,2} & =\left(\frac{2 \pi}{3}\right)^{1 / 2} e^{-\pi i / 12}, & \gamma_{2,2} & =\left(\frac{2 \pi}{3}\right)^{1 / 2} e^{7 \pi i / 12} .
\end{aligned}
$$

The formula (6.1), together with the Bessel function relations

$$
\frac{d}{d z}\left\{z^{ \pm \mu} J_{\mu}(z)\right\}= \pm z^{ \pm \mu} J_{\mu \mp 1}(z)
$$

show that

$$
v_{-1}^{\prime}(x)=\frac{d}{d x}\left\{\Psi(x) \xi^{1 / 3}\left(\gamma_{1,1} J_{-1 / 3}(\xi)+\gamma_{2,1} J_{1 / 3}(\xi)\right)\right\}+\frac{B(x, \lambda)}{\lambda^{1 / 3}} .
$$

The forms (6.2), (6.7) and (6.4) of $v_{-1}(x)$ are set forth, together with the forms of other solutions yet to be derived, respectively in (7.5c), (7.5d) and (7.5e) below.

A comparison of the formulas (6.3) and (6.6) with the respective ones (6.2) and (6.4), show that on the intervals on which $|\xi|>N$ the form for the 
derivative is formally obtainable from that of the solution itself by the systematic replacement of $e^{i \xi}[\sigma]$ by $i \lambda e^{i \xi}[\sigma \phi]$, for any $\sigma$, and of $e^{-i \xi}[\sigma]$ by $-i \lambda e^{-i \xi}[\sigma \phi]$. A comparison of the formulas (6.9) and (6.7) shows correspondingly that on an interval on which $|\xi| \leqq N$ the differentiation is formally carried out by actually differentiating the explicit part of the formula and multiplying the remainder term by $\lambda^{2 / 3}$. These observations can be shown to apply to all the solutions that will be discussed. We shall therefore omit, in the continuing discussion, the especial mention of the derivatives.

When $x>x_{1}$ and $0 \leqq \arg \lambda \leqq \pi-\epsilon$, so that $\xi$ lies in the sector $\Xi^{(0)}$, and likewise when $\epsilon \leqq \arg \lambda<\pi$, so that $\xi$ is in $\Xi^{(1)}$, the differential equation has a solution $\left({ }^{5}\right)$ of the form

$$
v_{-2}(x)=\frac{1}{\lambda^{1 / 6} \phi^{1 / 2}(x)} e^{i \xi}[1] .
$$

The form of this solution (of these solutions) when $x$ is on the interval $\left(-x_{0}, x_{0}\right)$ is

$$
v_{-2}(x)=\frac{1}{\lambda^{1 / 6} \phi^{1 / 2}(x)}\left\{e^{i \xi(x)}[1]+e^{-i \xi(x)}[i]\right\} .
$$

These formulas are included in the sets given below in (7.5e) and (7.5c). The form of $v_{-2}(x)$ in the intermediate interval $\left(x_{0}, x_{1}\right)$ is included in $(7.5 \mathrm{~d})$.

The Wronskian of any pair of solutions of the differential equation (4.4) is independent of $x$. From the formulas (7.5c), and the respective ones for the derivatives, it is found that

$$
W\left(v_{-1}, v_{-2}\right)=2 i \lambda^{2 / 3}[1] .
$$

The solutions $v_{-1}(x)$ and $v_{-2}(x)$ are thus linearly independent, at least when $|\lambda|$ is sufficiently large.

7. Completion of the forms of the $v_{-j}(x)$. Because the coefficient $\phi^{2}(x)$ of the differential Equation (4.4) is even in $x$, the functions $v_{-1}(-x)$ and $v_{-2}(-x)$ are also solutions. Linear relations

$$
v_{-j}(x)=c_{1, j} v_{-1}(-x)+c_{2, j} v_{-2}(x)
$$$$
j=1,2
$$

with coefficients that are independent of $x$ accordingly maintain. Now when $x$ is on the sub-interval $\left(-x_{0}, x_{0}\right)$ the same is true of $-x$. Therefore the formulas (7.5c) remain valid when $x$ is replaced by $-x$, and can be used for each of the functions that appear in the relations (7.1). These relations are thereupon expressible in terms of $e^{i \xi(x)}$ and $e^{-i \xi(x)}$, by use of the evaluation

$$
i \xi(-x)=\pi \lambda / 2-i \xi(x),
$$

(5) It may not be concluded that the solution in question is the same one for the two ranges of $\arg \lambda$. However, we find the single designation $v_{-2}(x)$ adequate. The solutions are those that are denoted by $u_{0,1}(z)$ and $u_{1.1}(z)$ in $[4,(21)]$. 
which is derivable from the definitions (2.1) and (5.1). A comparison of the coefficients of like exponentials then yields the evaluations

$$
\begin{array}{ll}
c_{1,1}=-i e^{-\pi \lambda / 2}[1], & c_{2,1}=e^{-\pi \lambda / 2}[1], \\
c_{1,2}=e^{\pi \lambda / 2}[1]+e^{-\pi \lambda / 2}[1], & c_{2,2}=i e^{-\pi \lambda / 2}[1] .
\end{array}
$$

Herewith the relations (7.1) give the forms of the functions $v_{-1}(x)$ and $v_{-2}(x)$ for $x<-x_{1}$ in terms of those already set down in (7.5e), and for $-x_{1} \leqq x \leqq-x_{0}$ in terms of those in (7.5d). These forms are set forth in (7.5a) and (7.5b) below, with the use of coefficients $\beta_{1, j}$, and $\beta_{2, j}$, defined by the relations

$$
\begin{aligned}
& \beta_{1, j}=c_{1, j} y_{1,1}+c_{2, j} \gamma_{1,2}, \\
& \beta_{2, j}=c_{1, j} \gamma_{2,1}+c_{2, j} \gamma_{2,2},
\end{aligned} \quad j=1,2 .
$$

We shall adopt the designations

$$
v_{1}(x)=v_{-1}(-x), \quad v_{2}(x)=v_{-2}(-x) .
$$

The forms of these solutions, the relation (7.2) having been used in their deduction, are likewise recorded in the sets of formulas (7.5). These latter are then as follows:

For $x<-x_{1}$,

$$
\begin{array}{rl}
v_{1}(x) & =\lambda^{-1 / 6} \phi^{-1 / 2}(x)\left\{e^{-\pi \lambda / 2+i \xi(x)}[1]+e^{\pi \lambda / 2-i \xi(x)}[\delta]\right\}, \\
v_{2}(x) & =\lambda^{-1 / 6} \phi^{-1 / 2}(x) e^{\pi \lambda / 2-i \xi(x)}[1], \\
v_{-j}(x) & =\lambda^{-1 / 6} \phi^{-1 / 2}(x)\left\{c_{1, j}[1] e^{-\pi \lambda / 2+i \xi(x)}+\left(c_{1, j}[\delta]+c_{2, j}[1]\right) e^{\pi \lambda 2-i \xi(x)}\right\}, \\
j & j=1,2 .
\end{array}
$$

For $-x_{1} \leqq x \leqq-x_{0}$,

$$
\begin{aligned}
v_{j}(x)= & \Psi(-x) \xi^{1 / 3}(-x)\left\{\gamma_{1, j} J_{-1 / 3}(\xi(-x))+\gamma_{2, j} J_{1 / 3}(\xi(-x))\right\}+\frac{B(x, \lambda)}{\lambda} \\
v_{-j}(x)= & \Psi(-x) \xi^{1 / 3}(-x)\left\{\beta_{1, j} J_{-1 / 3}(\xi(-x))+\beta_{2, j} J_{1 / 3}(\xi(-x))\right\} \\
& +\frac{c_{1, j} B+c_{2, j} B}{\lambda}
\end{aligned}
$$

For $-x_{0}<x<x_{0}$,

$$
\begin{aligned}
v_{1}(x) & =\lambda^{-1 / 6} \phi^{-1 / 2}(x) e^{-\pi \lambda / 2+i \xi(x)}[1] \\
v_{2}(x) & =\lambda^{-1 / 6} \phi^{-1 / 2}(x)\left\{e^{-\pi \lambda / 2+i \xi(x)}[i]+e^{\pi \lambda / 2-i \xi(x)}[1]\right\} \\
v_{-1}(x) & =\lambda^{-1 / 6} \phi^{-1 / 2}(x) e^{-i \xi(x)}[1] \\
v_{-2}(x) & =\lambda^{-1 / 6} \phi^{-1 / 2}(x)\left\{e^{i \xi(x)}[1]+e^{-i \xi(x)}[i]\right\}
\end{aligned}
$$

For $x_{0} \leqq x \leqq x_{1}$, 


$$
v_{j}(x)=\Psi(x) \xi^{1 / 3}(x)\left\{\beta_{1, j} J_{-1 / 3}(\xi(x))+\beta_{2, j} J_{1 / 3}(\xi(x))\right\}+\frac{c_{1, j} B+c_{2, j} B}{\lambda},
$$

$$
v_{-j}(x)=\Psi(x) \xi^{1 / 3}(x)\left\{\gamma_{1, j} J_{-1 / 3}(\xi(x))+\gamma_{2, j} J_{1 / 3}(\xi(x))\right\}+\frac{B(x, \lambda)}{\lambda} .
$$

For $x_{1}<x$,

$$
\begin{aligned}
v_{j}(x) & =\lambda^{-1 / 6} \phi^{-1 / 2}(x)\left\{\left(c_{1, j}[\delta]+c_{2, j}[1]\right) e^{i \xi(x)}+c_{1, j}[1] e^{-i \xi(x)}\right\}, \\
(7.5 \mathrm{e}) v_{-1}(x) & =\lambda^{-1 / 6} \phi^{-1 / 2}(x)\left\{e^{i \xi(x)}[\delta]+e^{-i \xi(x)}[1]\right\}, \\
v_{-2}(x) & =\lambda^{-1 / 6} \phi^{-1 / 2}(x) e^{i \xi(x)}[1] .
\end{aligned}
$$

The relations (7.1) and (7.3) lead from the Wronskian evaluation (6.10) to the further ones

$$
\begin{aligned}
W\left(v_{1}, v_{2}\right) & =-2 i \lambda^{2 / 3}[1], \\
W\left(v_{1}, v_{-1}\right) & =-2 i \lambda^{2 / 3} e^{-\pi \lambda / 2}[1], \\
W\left(v_{2}, v_{-1}\right) & =2 \lambda^{2 / 3} e^{-\pi \lambda / 2}[1] . \\
W\left(v_{2}, v_{-2}\right) & =2 i \lambda^{2 / 3}\left\{e^{\pi \lambda / 2}[1]+e^{-\pi \lambda / 2}[1]\right\} .
\end{aligned}
$$

It was remarked in $\S 6$ that the solutions of the differential equation (4.4) whose forms were to be determined are particular ones. Their particularity is now observable. By the rotation of the Fig. 1 through a right angle the locus of the values $i \xi(x)$ is obtainable.

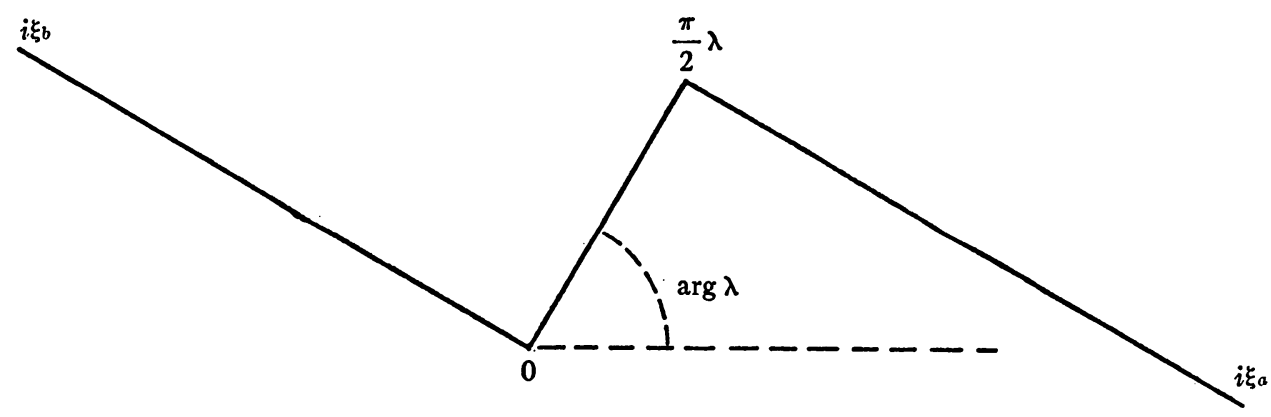

FIG. 2

This is shown in Fig. 2 when $0 \leqq \arg \lambda<\pi / 2$, and in Fig. 3 when $\pi / 2 \leqq \arg \lambda<\pi$. From these figures and the formulas (7.5a), (7.5c) and (7.5e) it can be seen that, at least outside of the turning-point neighborhoods, the function $v_{1}(x)$ has an absolute value which tends to a minimum at, or near, a turning point, and is monotonically increasing therefrom on either side. The turning point in question is $x=1$ when $0 \leqq \arg \lambda<\pi / 2$, and $x=-1$ when $\pi / 2 \leqq \arg \lambda<\pi$. The function $v_{-1}(x)$ is similarly related to the other turning point. The func- 


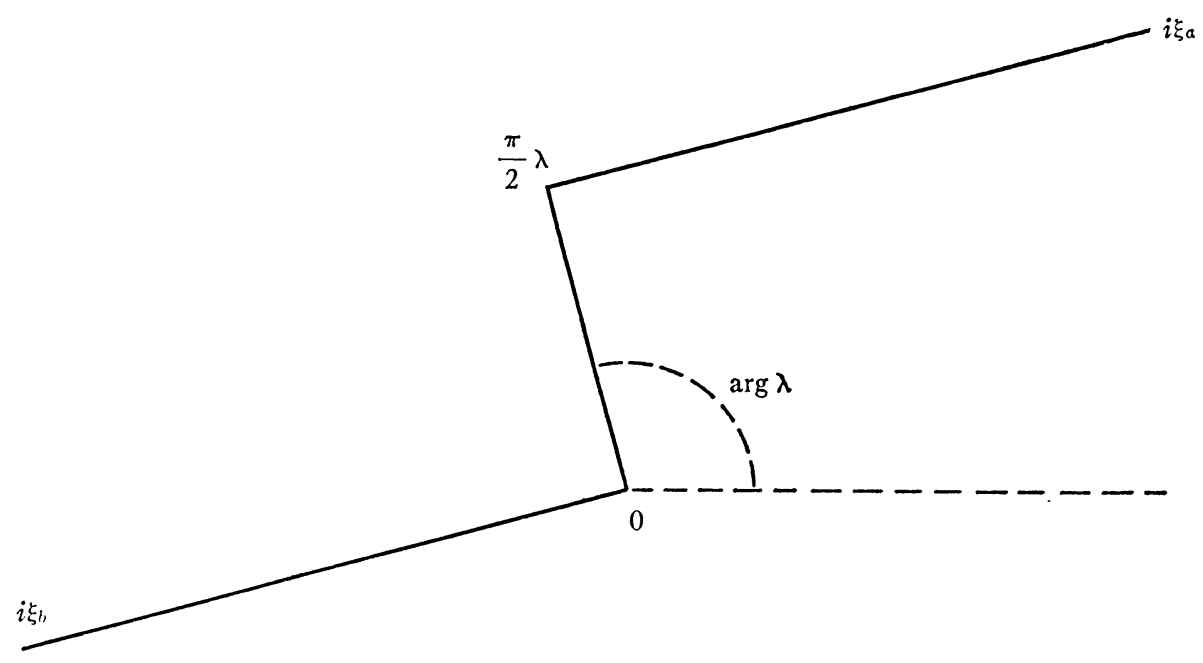

FIG. 3

tion $v_{2}(x)$ is nondecreasing in absolute value at least for all $x$ for which $|\xi(|x|)|>N$ and for all such the modulus of $v_{-2}(x)$ is nonincreasing.

8. The integral equation. If $u(x)$ is any function which fulfills the given Equation (4.2), the differential equation

$$
u^{\prime \prime}+\lambda^{2} \phi^{2}(x) u=-g(x, \lambda) u(x)
$$

is a nonhomogeneous one whose reduced associate is the related equation (4.4). The solutions of this Equation (8.1) are therefore expressible in terms of the functions $v(x)$ along with $g(x, \lambda) u(x)$, the method of "variation of constants" showing it to be of the form

$$
v(x)+\frac{1}{\lambda} \int_{x_{c}}^{x} Q(x, t, \lambda) u(t) d t .
$$

In this $x_{c}$ is any convenient limit of integration, a change in which can be compensated for by a change in the choice of $v(x)$, while $Q(x, t, \lambda)$ is given by the formula

$$
Q(x, t, \lambda)=\left\{\frac{v_{l}(x) v_{m}(t)-v_{m}(x) v_{l}(t)}{W\left(v_{l}, v_{m}\right)}\right\} \lambda g(t, \lambda) .
$$

It is readily seen that the Formula (8.2) is independent of the choice of $v_{l}(x)$ and $v_{m}(x)$ as solutions of the Equation (4.4), although to give a meaningful result these solutions must be linearly independent. Now the function $u(x)$ originally referred to is itself a solution of the Equation (8.1). The observations made, therefore apply to it, and show that it fulfills an equation 


$$
u(x)=v(x)+\frac{1}{\lambda} \int_{x_{c}}^{x} Q(x, t, \lambda) u(t) d t .
$$

Every solution of the differential Equation (4.1) is thus also a solution of the integral Equation (8.3) with an appropriate solution of the Equation (4.4) as $v(x)$.

The converse of this can also be proved, namely, if $x_{c}$ is chosen in any way, and $v(x)$ is taken to be any specific solution of the Equation (4.4), the solution of the resulting integral Equation (8.3) is a solution of the differential Equation (4.2). We shall, in accordance with this fact, use the Equation (8.3), with appropriate choices of $x_{c}$ and $v(x)$ as a source of solutions of the differential equation whose forms the Equation (8.3) permits us to determine.

Since the function $Q(x, t, \lambda)$ vanishes when $t=x$, the Equation (8.3) differentiates into

$$
u^{\prime}(x)=v^{\prime}(x)+\frac{1}{\lambda} \int_{x_{c}}^{x} \frac{\partial}{\partial x} Q(x, t, \lambda) u(t) d t .
$$

This is a formula for $u^{\prime}(x)$ which can be used when the form of $u(x)$ has been determined.

The following lemma will be of use in connection with the Equation (8.3).

LemmA. If, when $x_{*}$ and $x$ are on some specified interval and $|\lambda|$ is sufficiently large, the equation

$$
U(x)=F(x, \lambda)+\frac{1}{\lambda} \int_{x_{*}}^{x} K(x, t, \lambda) U(t) d t
$$

is one for which the conditions

$$
\begin{array}{rr}
\text { (a) }\left|\int_{x_{*}}^{x}\right| K(x, t, \lambda)|d t| & \leqq M, \\
\text { (b) } \quad|F(x, \lambda)| \leqq M,
\end{array}
$$

are fulfilled, $M$ being independent of $x$ and $\lambda$, the equation has a solution of the form

$$
U(x)=F(x, \lambda)+\frac{B(x, \lambda)}{\lambda} .
$$

The conditions (8.6) insure the uniform convergence of the series that is obtained by the successive iteration of the Equation (8.5). From that the proof of the lemma follows.

9. A solution $u_{-1}(x)$ or $u_{1}(x)$ when $x<-x_{0}$. It was observed at the close of $\S 7$ that, broadly speaking $v_{-1}(x)$ takes on its minimum absolute value at the turning point $x=-1$ when $\lambda$ is in the first quadrant of the complex plane, 
whereas $v_{1}(x)$ does so when $\lambda$ is in the second quadrant. We shall begin by considering the Equation (8.3) with $x_{c}=-1$, and with the role of $v(x)$ filled by this function of minimum modulus. To adapt a single deduction to the two $\lambda$-quadrants we shall resort to the use of double signs. It is therefore to be agreed, and the agreement is to be held to throughout the ensuing analysis, that a double sign is in every instance to be understood to signify its upper one when $0 \leqq \arg \lambda<\pi / 2$, and its lower one when $\pi / 2 \leqq \arg \lambda<\pi$. The integral equation to be considered is, therefore,

$$
u_{\mp 1}(x)=v_{\mp 1}(x)+\frac{1}{\lambda} \int_{-1}^{x} Q(x, t, \lambda) u_{\mp 1}(t) d t .
$$

We begin by considering this equation for $x$ in the sub-interval which contains the point $x=-1$. To this the formulas (7.5b) apply. The coefficients $\gamma_{1,1}$ and $\gamma_{2,1}$ are independent of $\lambda$, whereas, by (7.3) and (7.4) $\beta_{1,1}$ and $\beta_{2,1}$ contain $e^{-\pi \lambda / 2}$ as a factor. Thus $v_{\mp 1}(x)$ is of the form $e^{-\pi \lambda / 4 \mp_{\pi \lambda / 4}} B(x, \lambda)$. We shall use the reciprocal of the exponential that appears in this as a multiplier of the Equation (9.1). After such multiplication this equation appears as

with

$$
U_{1,2}(x)=V_{1,2}(x)+\frac{1}{\lambda} \int_{-1}^{x} Q(x, t, \lambda) U_{1,2}(t) d t,
$$

$$
\begin{aligned}
& U_{1,2}(x)=e^{\pi \lambda / 4 \pm \pi \lambda / 4} u_{\mp 1}(x), \\
& V_{1,2}(x)=e^{\pi \lambda / 4 \pm \pi \lambda / 4} v_{\mp 1}(x) .
\end{aligned}
$$

By construction, then, the function $V_{1,2}(x)$ is bounded. Therefore the Equation (9.2) fulfills the condition (8.6b).

As to the kernel $Q(x, t, \lambda)$, we shall construct this by use of the formula (8.2) with $l=1$ and $m=2$. Since the evaluations (7.5b) apply to both the variables $x$ and $t$, it is seen that $Q(x, t, \lambda)$ is of the form $\lambda^{1 / 3} B(x, t, \lambda)$. Because the interval of integration is of a length whose order is that of $\lambda^{-2 / 3}$, the condition (8.6a) is fulfilled. We may therefore infer, by the lemma of $\S 8$ that

$$
U_{1,2}(x)=V_{1,2}(x)+\frac{B(x, \lambda)}{\lambda} \text {. }
$$

By virtue of (9.3) this gives

$$
u_{\mp 1}(x)=v_{\mp 1}(x)+\frac{e^{-\pi \lambda / 4 \mp \pi \lambda / 4} B(x, \lambda)}{\lambda}, \quad \text { for }-x_{1} \leqq x \leqq-x_{0} .
$$

It is to be observed that this conclusion implies that, to the extent of their explicitness, the formulas given in $(7.5 \mathrm{~b})$ for the functions $v_{\mp 1}(x)$ apply also to the respective solutions $u_{\mp 1}(x)$. For, an expression of the form

$$
[1]+B(x, \lambda) / \lambda
$$

is again of the form [1]. 
We next consider the Equation (9.1) for $x$ on the first sub-interval from the left, namely for $x$ on the interval $\left(-x_{a},-x_{1}\right)$. To this the evaluations (7.5a) apply. Inasmuch as the point $i \xi(x)$ now lies upon the right-hand segment of the broken line in Fig. 2 or Fig. 3, the vector $i \xi(x)-\pi \lambda / 2$ has a real component that is non-negative. Hence from (7.5a) the function $v_{\mp 1}(x)$ is of the form

$$
\lambda^{-1 / 6} \phi^{-1 / 2}(x) e^{-3 \pi \lambda / 4 \mp \pi \lambda / 4+i \xi(x)} B(x, \lambda) .
$$

With the reciprocal of the coefficient of $B(x, \lambda)$ in this as a multiplier, the Equation (9.1) is given the form

$$
U_{1,1}(x)=F_{1,1}(x, \lambda)+\frac{1}{\lambda} \int_{-x_{1}}^{x} K_{-1}(x, t, \lambda) U_{1,1}(t) d t,
$$

with

$$
\begin{aligned}
& U_{1,1}(x)=\lambda^{1 / 6} \phi^{1 / 2}(x) e^{3 \pi \lambda / 4 \pm \pi \lambda / 4-i \xi(x)} u_{\mp 1}(x), \\
& V_{1,1}(x)=\lambda^{1 / 6} \phi^{1 / 2}(x) e^{3 \pi \lambda / 4 \pm \pi \lambda / 4-i \xi(x)} v_{\mp 1}(x),
\end{aligned}
$$

and with

$$
K_{\mp 1}(x, t, \lambda)=\left\{\frac{\phi(x)}{\phi(t)}\right\}^{1 / 2} e^{\mp i \xi(x) \pm i \xi(t)} Q(x, t, \lambda),
$$

and

$$
F_{1,1}(x, \lambda)=V_{1,1}(x)+\frac{1}{\lambda} \int_{-1}^{-x_{1}} K_{-1}(x, t, \lambda) U_{1,1}(t) d t .
$$

We write the equation in the manner (9.5), (9.8), because for $t$ between -1 and $-x_{1}$ the form of $U_{1,1}(t)$ is already known, inasmuch as it was observed to be obtainable from the same formula as gives $V_{1,1}(t)$.

For use in connection with the Equation (9.5) and the formula (9.8) we draw from the evaluations (7.5a) and (7.5b) those of the following Table $1_{1}$.

\begin{tabular}{c|c|c}
$t$-interval & $\left(-x_{a},-x_{1}\right)$ & $\left(-x_{1},-x_{0}\right)$ \\
\hline$Q(x, t, \lambda)$ & $\frac{e^{i \xi(x)-i \xi(t)} B(x, t, \lambda)}{\phi^{1 / 2}(x) \phi^{1 / 2}(t)}$ & $\frac{e^{-\pi \lambda / 2+i \xi(x)} B(x, t, \lambda)}{\lambda^{1 / 6} \phi^{1 / 2}(x)}$ \\
\hline$U_{1,1}(t)$ & & $\lambda^{1 / 6} \phi^{1 / 2}(x) e^{\pi / 2-i \xi(t)} B(t)$ \\
\hline
\end{tabular}


From the first entry that appears in this for $Q(x, t, \lambda)$, together with the relation (9.7), it is to be seen that $K_{-1}(x, t, \lambda)$ is of the form $B(x, t, \lambda) / \phi(t)$. Since the integral of $1 /|\phi(t)|$ exists and is bounded, even in a neighborhood of the point $t=-1$, the Equation (9.5) is seen to fulfill the condition (8.6a).

The relation (9.7), together with the entries in the table appropriate to the $t$-interval $\left(-x_{1},-x_{0}\right)$ show that the integrand in the formula (9.8) is bounded. Hence $F_{1,1}(x, \lambda)$ differs only by an amount $B(x, \lambda) / \lambda$ from its term $V_{1,1}(x)$, and since this latter is bounded, by construction, the Equation (9.5) also fulfills the condition (8.6b). Accordingly

$$
U_{1,1}(x)=F_{1,1}(x, \lambda)+\frac{B(x, \lambda)}{\lambda},
$$

and this relation continues to be valid if $F_{1,1}(x, \lambda)$ is replaced by $V_{1,1}(x)$. Thus, by (9.6)

$$
u_{\mp 1}(x)=v_{\mp 1}(x)+\frac{e^{-3 \pi \lambda / 4 \mp \pi \lambda / 4+i \xi(x)} B(x, \lambda)}{\lambda^{7 / 6} \phi^{1 / 2}(x)}, \quad \text { for }-x_{a} \leqq x<-x_{1} .
$$

It will be observed again, that, to the extent of their explicitness, the formulas (7.5a) for $v_{\mp 1}(x)$ also apply to $u_{\mp 1}(x)$.

10. The solution $u_{\mp 1}(x)$ on the interval $\left(-x_{0}, x_{b}\right)$. When $x$ is on the third sub-interval from the left, namely on $\left(-x_{0}, x_{0}\right)$ we observe from the formulas (7.5c) that $V_{1,3}(x)$ is bounded if

$$
\begin{aligned}
& U_{1,3}(x)=\lambda^{1 / 6} \phi^{1 / 2}(x) e^{\pi \lambda / 4 \mp \pi \lambda / 4 \pm i \xi(x)} u_{\mp 1}(x), \\
& V_{1,3}(x)=\lambda^{1 / 6} \phi^{1 / 2}(x) e^{\pi \lambda / 4 \mp \pi \lambda / 4 \pm i \xi(x)} v_{\mp 1}(x) .
\end{aligned}
$$

With the use of the appropriate multiplier the Equation (9.1) is put in to the form

$$
U_{1,3}(x)=F_{1,3}(x, \lambda)+\frac{1}{\lambda} \int_{-x_{0}}^{x} K_{ \pm 1}(x, t, \lambda) U_{1,3}(t) d t,
$$

with the kernel given by the Formula (9.7), and with

$$
F_{1,3}(x, \lambda)=V_{1,3}(x)+\frac{1}{\lambda} \int_{-1}^{-x_{0}} K_{ \pm 1}(x, t, \lambda) U_{1,3}(t) d t .
$$

For use in connection with the Equations (10.2) and (10.3), it is convenient to construct $Q(x, t, \lambda)$ by the use of the Formula (8.2) with $l=1$ and $m=2$ when $-1 \leqq t \leqq-x_{0}$, and with $l= \pm 1$ and $m=2$ when $-x_{0}<t<x_{0}$. For the $x$-values now under consideration, the point $i \xi(x)$ lies on the central segment of the broken line of Fig. 2 or Fig. 3. The real components of the vectors $\pi \lambda / 2-i \xi(x)$ and $-i \xi(x)+i \xi(t)$ are therefore positive when Fig. 2 applies and negative when Fig. 3 applies. With an eye to the dominant terms involved, we therefore obtain from the Formulas (7.5b) and (7.5c) the evaluations of the 
Table $1_{3}$ with $U(t)$ signifying $U_{1,3}(t)$.

\begin{tabular}{c|c|c}
$t$-interval & $\left(-x_{1},-x_{0}\right)$ & $\left(-x_{0},-x_{0}\right)$ \\
\hline$Q(x, t, \lambda)$ & $\lambda^{1 / 6} \phi^{-1 / 2}(x) e^{ \pm \pi \lambda / 2 \mp i \xi(x)} B(x, t, \lambda)$ & $\phi^{-1 / 2}(x) \phi^{-1 / 2}(t) e^{\mp i \xi(x) \pm i \xi(t)}(B x, t, \lambda)$ \\
\hline$U(t)$ & $\lambda^{1 / 6} \phi^{1 / 2}(t) e^{\mp \pi \lambda / 2 \pm i \xi(t)} B(t, \lambda)$ & \\
\hline
\end{tabular}

\section{TABLE $1_{3}$}

From these it can be seen that the Equation (10.2) fulfills the conditions (8.6a) and (8.6b), and hence that

$$
U_{1,3}(x)=V_{1,3}(x)+\frac{B(x, \lambda)}{\lambda} .
$$

By virtue of (10.1) this yields

$$
u_{\mp 1}(x)=v_{\mp 1}(x)+\frac{e^{-\pi \lambda / 4 \pm \pi \lambda / 4 \mp i \xi(x)} B(x, \lambda)}{\lambda^{7 / 6} \phi^{1 / 2}(x)}, \quad \text { for }-x_{0}<x<x_{0} .
$$

When $x$ is on the interval $\left(x_{0}, x_{1}\right)$ the procedure that is now familiar leads to the expression of the Equation (9.1) in the form

$$
U_{1,4}(x)=F_{1,4}(x, \lambda)+\frac{1}{\lambda} \int_{x_{0}}^{x} Q(x, t, \lambda) U_{1,4}(t) d t,
$$

with

and

$$
U_{1,4}(x)=e^{\pi \lambda / 4 \mp \pi \lambda / 4} u_{\mp 1}(x), \quad V_{1,4}(x)=e^{\pi \lambda / 4 \mp \pi \lambda / 4} v_{\mp 1}(x),
$$

$$
F_{1,4}(x, \lambda)=V_{1,4}(x)+\frac{1}{\lambda} \int_{-1}^{x_{0}} Q(x, t, \lambda) U_{1,4}(t) d t .
$$

The construction of $Q(x, t, \lambda)$ from the functions $v_{-1}(x)$ and $v_{-2}(x)$ yields the evaluations of Table $1_{4}$ with $U(t)$ signifying $U_{1,4}(t)$.

\begin{tabular}{c|c|c|c}
$t$-interval & $\left(-x_{1},-x_{0}\right)$ & $\left(-x_{0}, x_{0}\right)$ & $\left(x_{0}, x_{1}\right)$ \\
\hline$Q(x, t, \lambda)$ & $\lambda^{1 / 3} e^{ \pm \pi \lambda / 2} B(x, t, \lambda)$ & $\lambda^{1 / 6} \phi^{-1 / 2}(t) e^{ \pm i \xi(t)} B(x, t, \lambda)$ & $\lambda^{1 / 3} B(x, t, \lambda)$ \\
\hline$U(t)$ & $e^{\mp \pi \lambda / 2} B(t, \lambda)$ & $\lambda^{-1 / 6} \phi^{-1 / 2}(t) e^{\mp i \xi(t)} B(t, \lambda)$ & \\
\hline
\end{tabular}


From these it is found that the Equation (10.5) fulfills the conditions of the lemma of $\S 8$ and thus that

$$
u_{\mp 1}(x)=v_{\mp 1}(x)+\frac{e^{-\pi \lambda / 4 \pm \pi \lambda / 4} B(x, \lambda)}{\lambda}, \quad \text { for } x_{0} \leqq x \leqq x_{1} .
$$

Finally, when $x$ is on the interval $\left(x_{1}, x_{b}\right)$ we write the Equation (9.1) in the form

$$
U_{1,5}(x)=F_{1,5}(x, \lambda)+\frac{1}{\lambda} \int_{x_{1}}^{x} K_{1}(x, t, \lambda) U_{1,6}(t) d t
$$

with

$$
\begin{aligned}
& U_{1,5}(x)=e^{\pi \lambda / 4 \mp \pi \lambda / 4+i \xi(x)} u_{\mp 1}(x), \\
& V_{1,5}(x)=e^{\pi \lambda / 4 \mp \pi \lambda / 4+i \xi(x)} x_{\mp 1}(x),
\end{aligned}
$$

and

$$
F_{1,5}(x, \lambda)=V_{1,5}(x)+\frac{1}{\lambda} \int_{-1}^{x_{1}} K_{1}(x, t, \lambda) U_{1,5}(t) d t .
$$

The appropriate table for use in connection with this is Table $1_{5}$ with

\begin{tabular}{c|c|c|c|c}
$t$-interval & $\left(-x_{1},-x_{0}\right)$ & $\left(-x_{0}, x_{0}\right)$ & $\left(x_{0}, x_{1}\right)$ & $\left(x_{1}, x_{b}\right)$ \\
\hline$Q(x, t, \lambda)$ & $\frac{\lambda^{1 / 6} e^{ \pm \pi \lambda / 2-i \xi(x)} B(x, t, \lambda)}{\phi^{1 / 2}(x)}$ & $\frac{e^{-i \xi(x) \pm i \xi(t)} B(x, t, \lambda)}{\phi^{1 / 2}(x) \phi^{1 / 2}(t)}$ & $\frac{\lambda^{1 / 6} e^{-i \xi(x)} B(x, t, \lambda)}{\phi^{1 / 2}(x)}$ & $\frac{e^{-i \xi(x)+i \xi(t)} B(x, t, \lambda)}{\phi^{1 / 2}(x) \phi^{1 / 2}(t)}$ \\
\hline$U(t)$ & $-\lambda^{1 / 6} \phi^{1 / 2}(t) e^{\mp \pi \lambda / 2+i \xi(t)} B(t, \lambda)$ & $e^{\mp i \xi(t)+i \xi(t)} B(t, \lambda)$ & $\lambda^{1 / 6} \phi^{1 / 2}(t) e^{i \xi(t)} B(t, \lambda)$ & \\
\hline
\end{tabular}

\section{TABLE $1_{5}$}

$U(t)$ taken to be $U_{1,5}(t)$. The conclusion is

$$
u_{\mp 1}(x)=v_{\mp 1}(x)+\frac{e^{-\pi \lambda / 4 \pm \pi \lambda / 4-i \xi(x)} B(x, \lambda)}{\lambda^{7 / 6} \phi^{1 / 2}(x)}, \quad \text { for } x_{1}<x \leqq x_{b} .
$$

We observe that in each instance, (10.4), (10.6), and (10.7), the formula for $v_{\mp 1}(x)$ serves, to the extent of its explicitness, to give also the form of $u_{\mp 1}(x)$.

11. Solutions $y_{1}(s)$ and $y_{-1}(s)$ of the given differential equation. The analyses of $\S 9$ and $\S 10$ can be adapted, in effect by the systematic interchange of $x$ and $t$ with $-x$ and $-t$ to apply to the integral equation

$$
u_{ \pm 1}(x)=v_{ \pm 1}(x)+\frac{1}{\lambda} \int_{1}^{x} Q(x, t, \lambda) u_{ \pm 1}(t) d t .
$$


By that means the forms of solutions $u_{1}(x)$ and $u_{-1}(x)$ are obtainable for those values of $\arg \lambda$ for which $\S 9$ and $\S 10$ dealt respectively with $u_{-1}(x)$ and $u_{1}(x)$. The conclusion so reached is that the formulas (7.5) for $v_{ \pm 1}(x)$ are, to the extent of their explicitness, also descriptive of the respective solutions $u_{ \pm 1}(x)$.

The functions $u_{ \pm 1}(x)$ are solutions of the given differential equation in its normalized form (4.2). In its original form (1.1) the equation is solved by functions $y(s)$ which, by (4.1) and (2.4) are obtainable from the relation

$$
y(s)=\left\{\frac{\phi(x)}{\theta(s)}\right\}^{1 / 2} u(x)
$$

with $s$ and $x$ related by the implicit Equation (2.3). At the same time we observe from (4.3) that the functions $v_{\mp 1}(x)$ are, in fact, the same as

$$
w_{\mp 1}\left((i \lambda)^{1 / 2} x, \quad(i \lambda-1) / 2\right),
$$

where $w_{-1}(z, \kappa)$ and $w_{1}(z, \kappa)$ are solutions of the Weber Equation (1.2).

Let the functions $\xi_{j}(s)$ and $\Psi_{j}(s)$ for $j=1,2$, be given by the formulas

$$
\begin{aligned}
\xi_{1}(s) & =\lambda \int_{\beta}^{s} \theta(s) d s, & \xi_{2}(s) & =\lambda \int_{s}^{\alpha} \theta(s) d s, \\
\Psi_{1}(s) & =\frac{\left\{\int_{\beta}^{s} \theta(s) d s\right\}^{1 / 6}}{\theta^{1 / 2}(s)}, & \Psi_{2}(s) & =\frac{\left\{\int_{s}^{\alpha} \theta(s) d s\right\}^{1 / 6}}{\theta^{1 / 2}(s)}
\end{aligned}
$$

with $\Psi_{1}(s)$ and $\Psi_{2}(s)$ defined to be continuous respectively at $s=\beta$ and $s=\alpha$. By the relations (2.3), (2.3a) and (5.1), then,

$$
\begin{array}{rlrl}
\xi(x) & \equiv \xi_{1}(s), & \xi(-x) & \equiv \xi_{2}(s), \\
\left\{\frac{\phi(x)}{\theta(s)}\right\}^{1 / 2} \Psi(x) & \equiv \Psi_{1}(s), \quad\left\{\frac{\phi(x)}{\theta(s)}\right\}^{1 / 2} \Psi(-x) \equiv \Psi_{2}(s) .
\end{array}
$$

With these evaluations at hand, and by virtue of the fact, which follows from (6.8), (7.3) and (7.4), that

$$
\beta_{1,1}=-e^{-\pi \lambda / 2}\left[\gamma_{2,2}\right], \quad \beta_{2,1}=-e^{-\pi \lambda / 2}\left[\gamma_{1,2}\right],
$$

we may summarize the results of $\S 9$ and $\S 10$ as follows:

TheOREM 1. The differential Equation (1.1) has, both when $0 \leqq \arg \lambda<\pi / 2$, and when $\pi / 2 \leqq \arg \lambda<\pi$, a pair of solutions $y_{1}(s)$ and $y_{-1}(s)$ which are asymptotically representable over the whole given interval $a \leqq s \leqq b$ in the manner

$$
y_{\mp 1}(s) \sim\left\{\frac{x^{2}(s)-1}{\theta^{2}(s)}\right\}^{1 / 4} w_{\mp 1}\left((i \lambda)^{1 / 2} x(s),(i \lambda-1) / 2\right),
$$


where $w_{-1}(z, \kappa)$ and $w_{1}(z, \kappa)$ are Weber functions. In consequence of this the solutions $y_{1}(s)$ and $y_{-1}(s)$ have in the several parts of the interval $(a, b)$ the following forms:

For $a \leqq s<\alpha$ and $\left|\xi_{2}(s)\right|>N$,

$$
\begin{aligned}
y_{1}(s) & =\Psi_{2}(s) \xi_{2}^{-1 / 6}(s)\left\{e^{-i \xi_{2}(s)}[1]+e^{i \xi_{2}(s)}[\delta]\right\} \\
y_{-1}(s) & =\Psi_{2}(s) \xi_{2}^{-1 / 6}(s) e^{-\pi \lambda / 2}\left\{e^{-i \xi_{2}(s)}[-i]+e^{i \xi_{2}(s)}[1-\delta]\right\}
\end{aligned}
$$

For $\left|\xi_{2}(s)\right| \leqq N$,

$$
y_{1}(s)=\Psi_{2}(s) \xi_{2}^{1 / 3}(s)\left\{\gamma_{1,1} J_{-1 / 3}\left(\xi_{2}\right)+\gamma_{2,1} J_{1 / 3}\left(\xi_{2}\right)\right\}+\frac{B(s, \lambda)}{\lambda},
$$

$$
y_{-1}(s)=\Psi_{2}(s) \xi_{2}^{1 / 3}(s) e^{-\pi \lambda / 2}\left\{-\gamma_{2,2} J_{-1 / 3}\left(\xi_{2}\right)-\gamma_{1,2} J_{1 / 3}\left(\xi_{2}\right)\right\}+\frac{e^{-\pi \lambda / 2} B(s, \lambda)}{\lambda} .
$$

For $\alpha<s<\beta$, and $\left|\xi_{j}(s)\right|>N, j=1,2$,

$$
\begin{aligned}
y_{1}(s) & =\Psi_{l}(s) \xi_{l}^{-1 / 6}(s) e^{-i \xi_{2}(s)}[1], \\
y_{-1}(s) & =\Psi_{l}(s) \xi_{l}{ }^{-1 / 6}(s) e^{-i \xi_{1}(s)}[1], \quad \text { with } l \text { either } 1 \text { or } 2 .
\end{aligned}
$$

For $\left|\xi_{1}(s)\right| \leqq N$,

$$
y_{1}(s)=\Psi_{1}(s) \xi_{1}^{1 / 3}(s) e^{-\pi \lambda / 2}\left\{-\gamma_{2,2} J_{-1 / 3}\left(\xi_{1}\right)-y_{1,2} J_{1 / 3}\left(\xi_{1}\right)\right\}+\frac{e^{-\pi \lambda / 2} B(s, \lambda)}{\lambda},
$$

$$
y_{-1}(s)=\Psi_{1}(s) \xi_{1}^{1 / 3}(s)\left\{\gamma_{1,1} J_{-1 / 3}\left(\xi_{1}\right)+\gamma_{2,1} J_{1 / 3}\left(\xi_{1}\right)\right\}+\frac{B(s, \lambda)}{\lambda} .
$$

For $\beta<s \leqq b$, and $\left|\xi_{1}(s)\right|>N$,

$$
\begin{aligned}
y_{1}(s) & =\Psi_{1}(s) \xi_{1}^{-1 / 6}(s) e^{-\pi \lambda / 2}\left\{e^{i \xi_{1}(s)}[1-i \delta]+e^{-i \xi_{1}(s)}[-i]\right\}, \\
y_{-1}(s) & =\Psi_{1}(s) \xi_{1}^{-1 / 6}(s)\left\{e^{i \xi_{1}(s)}[\delta]+e^{-i \xi_{1}(s)}[1]\right\} .
\end{aligned}
$$

In these formulas $N$ is an adequately large constant; the coefficients $\delta$ and $\gamma_{l, j}$ are given by the Formulas (6.5) and (6.8); the notation $[\sigma]$ is explained in (5.4); and $B$ is a generic symbol for a function that is bounded.

The Formulas (11.5a), (11.5c) and (11.5e) are convertible into those for the respective derivatives by formally replacing $e^{i \xi_{i}}[\sigma]$ by $-(-1)^{i} i \lambda e^{i \xi_{j}}[\sigma \theta(s)]$, and $e^{-i \xi_{j}}[\sigma]$ by $(-1)^{i} i \lambda e^{-i \xi_{j}}[\sigma \theta(s)]$. The Formulas $(11.5 \mathrm{~b})$ and $(11.5 \mathrm{~d})$ are so convertible by differentiating their explicit parts and multiplying the remainder terms by $\lambda^{2 / 3}$.

12. A solution $u_{2}(x)$. The solutions $u_{1}(x)$ and $u_{-1}(x)$ are linearly independent, and on the central interval, $-x_{0}<x<x_{0}$, one is of increasing and the other of decreasing modulus, unless $\lambda$ is pure imaginary. On that interval, 
therefore, the independence of these solutions is clearly displayed by their asymptotic forms. That is not so on the end intervals $\left(-x_{a},-x_{1}\right)$ and $\left(x_{1}, x_{b}\right)$. For there, as may be seen from the formulas (7.5a) and (7.5e), both solutions are given by forms that involve a (the same) dominant exponential, unless $\lambda$ is real, and because of that neither of the solutions is distinguishable through this representation from a multiple of the other. This fact impairs the usefulness of this set of solutions on the sub-intervals in question, in fact makes it unsuitable for such analyses as bank with any degree of explicitness upon their functional independence. For that reason we turn to a consideration of other solutions $u_{2}(x)$ and $u_{-2}(x)$. Of these the former is to be obtained from the integral Equation (8.3) with $x_{c}$ and $v(x)$ respectively chosen to be $-x_{a}$ and $v_{2}(x)$. Thus

$$
u_{2}(x)=v_{2}(x)+\frac{1}{\lambda} \int_{-x_{a}}^{x} Q(x, t, \lambda) u_{2}(t) d t .
$$

When $x$ is on the interval $\left(-x_{a},-x_{1}\right)$ the multiplication of the Equation (12.1) by the appropriate factor gives it the form

$$
U_{2,1}(x)=V_{2,1}(x)+\frac{1}{\lambda} \int_{-x_{a}}^{x} K_{1}(x, t, \lambda) U_{2,1}(t) d t,
$$

with

$$
\begin{aligned}
& U_{2,1}(x)=\lambda^{1 / 6} \phi^{1 / 2} \phi(x) e^{-\pi \lambda / 2+i \xi(x)} u_{2}(x), \\
& V_{2,1}(x)=\lambda^{1 / 6} \phi^{1 / 2}(x) e^{-\pi \lambda / 2+i \xi(x)} v_{2}(x)
\end{aligned}
$$

The construction of $Q(x, t, \lambda)$ from the formula (8.2), with $l=1$ and $m=2$ shows, since $t \leqq x$, that

$$
Q(x, t, \lambda)=\phi^{-1 / 2}(x) \phi^{-1 / 2}(t) e^{-i \xi(x)+i \xi(t)} B(x, t, \lambda) .
$$

Since $V_{2,1}(x)$ is bounded, by construction, the Equation (12.2) fulfills the conditions (8.6). We may therefore draw from it that

$$
u_{2}(x)=v_{2}(x)+\frac{e^{\pi \lambda / 2-i \xi(x)} B(x, \lambda)}{\lambda^{7 / 6} \phi^{1 / 2}(x)}, \quad \text { for }-x_{a} \leqq x<-x_{1} .
$$

When $x$ is on the interval $\left(-x_{1},-x_{0}\right)$ we shall take the Equation (12.1) in the form

$$
u_{2}(x)=F_{2,2}(x, \lambda)+\frac{1}{\lambda} \int_{-x_{1}}^{x} Q(x, t, \lambda) u_{2}(t) d t
$$

with

$$
F_{2,2}(x, \lambda)=v_{2}(x)+\frac{1}{\lambda} \int_{-x_{a}}^{-x_{1}} Q(x, t, \lambda) u_{2}(t) d t .
$$


The entries of the Table $2_{2}$

\begin{tabular}{c|c|c|}
$(t$-interval & $\left(-x_{a},-x_{1}\right)$ & $\left(-x_{1},-x_{0}\right)$ \\
\hline$Q(x, t, \lambda)$ & $\lambda^{1 / 6} \phi^{-1 / 2}(t) e^{-\pi \lambda / 2+i \xi(t)} B(x, t, \lambda)$ & $\lambda^{1 / 3} B(x, t, \lambda)$ \\
\hline$u_{2}(t)$ & $\lambda^{-1 / 6} \phi^{-1 / 2}(t) e^{\pi \lambda / 2-i \xi(t)} B(t, \lambda)$ & \\
\hline
\end{tabular}

TABLE $22_{2}$

show that the lemma of $\$ 8$ applies, and that therefore

$$
u_{2}(x)=v_{2}(x)+\frac{B(x, \lambda)}{\lambda}, \quad \text { for }-x_{1} \leqq x \leqq-x_{0} .
$$

When $x$ is on the interval $\left(-x_{0}, x_{0}\right)$ the formula (7.5c) shows that the dominant exponential term of $v_{2}(x)$ is that which involves $e^{ \pm \pi \lambda / 2 \mp i \xi(x)}$, the ambiguous sign being again interpreted as was specified in $\S 9$. We therefore multiply the Equation (12.1) by the factor which permits its expression in the form

$$
U_{2,3}(x)=F_{2,3}(x, \lambda)+\frac{1}{\lambda} \int_{-x_{0}}^{x} K_{ \pm 1}(x, t, \lambda) U_{2,3}(t) d t
$$

with

$$
U_{2,3}(x)=\lambda^{1 / 6} \phi^{1 / 2}(x) e^{\mp \pi \lambda / 2 \pm i \xi(x)} u_{2}(x), \quad V_{2,3}(x)=\lambda^{1 / 6} \phi^{1 / 2}(x) e^{\mp \pi \lambda / 2 \pm i \xi(x)} v_{2}(x),
$$

and

$$
F_{2,3}(x, \lambda)=V_{2,3}(x)+\frac{1}{\lambda} \int_{-x_{a}}^{-x_{0}} K_{ \pm 1}(x, t, \lambda) U_{2,3}(t) d t .
$$

The table of evaluations appropriate for use with this is the Table $1_{3}$ of $\$ 10$ with $U(t)$ taken to be $U_{2,3}(t)$ and with an additional column, namely that of the Table $2_{3}$.

\begin{tabular}{c|c}
$t$-interval & $\left(-x_{a},-x_{1}\right)$ \\
\hline$Q(x, t, \lambda)$ & $\phi^{-1 / 2}(x) \phi^{-1 / 2}(t) e^{-\pi \lambda / 2 \pm \pi \lambda / 2 \mp i \xi(x)+i \xi(t)} B(x, t, \lambda)$ \\
\hline$U_{2,3}(t)$ & $e^{\pi \lambda / 2 \mp \pi \lambda / 2 \pm i \xi(t)-i \xi(t)} B(t, \lambda)$ \\
\hline
\end{tabular}


Therefrom it is found that

$$
u_{2}(x)=v_{2}(x)+\frac{e^{ \pm \pi \lambda / 2 \mp i \xi(x)}(B x, \lambda)}{\lambda^{7 / 6} \phi^{1 / 2}(x)}, \quad \text { for }-x_{0}<x<x_{0} .
$$

13. Characteristic values and solutions. With the choice of $x_{c}$ and $v(x)$ as the point $x_{b}$ and the function $v_{-2}(x)$ respectively, one obtains from (8.3) an equation whose solution we shall denote by $u_{-2}(x)$. The analysis of $\$ 12$ can effectively be adapted to this equation by the interchange of $-x_{a}$ with $x_{b}$ and the systematic interchange of $x$ and $t$ with $-x$ and $-t$ respectively. It is found in that way that the formulas $(7.5 \mathrm{e}),(7.5 \mathrm{~d})$, and $(7.5 \mathrm{c})$, for $v_{-2}(x)$ are, to the extent of their explicitness, also formulas for $u_{-2}(x)$.

From the forms of $u_{2}(x)$ and $u_{-2}(x)$ that have thus been deduced for the central interval $\left(-x_{0}, x_{0}\right)$ the Wronskian of these two solutions, which is, of course, independent of $x$, may be calculated. The formula so found is

$$
W\left(u_{2}, u_{-2}\right)=2 i \lambda^{2 / 3}\left\{e^{\pi \lambda / 2}[1]+e^{-\pi \lambda / 2}[1]\right\},
$$

and this clearly shows that for certain $\lambda$-values this Wronskian vanishes. These characteristic values are the roots of the respective equation-the characteristic equation

$$
e^{\pi \lambda / 2}[1]+e^{-\pi \lambda / 2}[1]=0,
$$

and as such their distribution is known, [5]. In the region $0 \leqq \arg \lambda<\pi$, and with $|\lambda|$ large, they are denumerably infinite. With appropriate successive integers $n$ they may, therefore, be denoted by $\lambda_{n}$. They are located then in accordance with a formula

$$
\lambda_{n}=(2 n+1) i+\epsilon_{n},
$$

in which $\epsilon_{n}$ is of the order of $1 / n$.

At a characteristic value the solutions $u_{2}(x)$ and $u_{-2}(x)$, which may be more explicitly designated by $u_{2}(x, \lambda)$ and $u_{-2}(x, \lambda)$, are thus linearly dependent. Since $e^{ \pm \pi \lambda_{n} / 2}=(-1)^{n}[1]$, as follows from (13.3), it may be seen from the formulas $(7.5 \mathrm{c})$ that

$$
u_{2}\left(x, \lambda_{n}\right) \equiv(-1)^{n}[1] u_{-2}\left(x, \lambda_{n}\right) .
$$

We shall call $u_{2}\left(x, \lambda_{n}\right)$ a characteristic solution of the differential Equation (4.2). Its form over the entire $x$-interval is at hand, for the forms of $u_{2}(x)$ and $u_{-2}(x)$ over the respective sub-intervals $\left(-x_{a}, x_{0}\right)$ and $\left(-x_{0}, x_{b}\right)$ have been determined, and are connected by the relation (13.4).

Since $\lambda_{n}^{2}$ is, at least asymptotically, real and negative, the characteristic solutions are oscillatory between the turning points. From the formulas (7.5c) their absolute values there are seen to be bounded as to $n$. Upon the transit of either turning point the solution departs from the oscillatory char- 
acter and assumes that of an exponential that decreases as $x$ recedes from the turning point. The characteristic solutions are thus uniformly bounded over the given $x$-interval.

By means of the relations (11.2), (11.3), and (11.4), the solution forms for the differential equation in its originally given form (1.1) can be inferred from those that have been derived for it in its normalized state (4.2). We obtain thus the following theorem.

Theorem 2. A differential Equation (1.1) admits for large values of $|\lambda| a$ set of characteristic values $\lambda_{n}$, whose distribution is given by a formula $\lambda_{n}$ $=(2 n+1) i+O(1 / n)$, with successive integral $n$. The respective characteristic solutions $y_{2}\left(s, \lambda_{n}\right)$ have the following forms:

For $a \leqq s<\alpha$, and $\left|\xi_{2}(s)\right|>N$,

$$
y_{2}\left(s, \lambda_{n}\right)=\Psi_{2}(s) \xi_{2}^{-1 / 6}(s) e^{i \xi_{2}(s)}[1] .
$$

For $\left|\xi_{2}(s)\right| \leqq N$,

$$
y_{2}\left(s, \lambda_{n}\right)=\Psi_{2}(s) \xi_{2}^{1 / 3}(s)\left\{\gamma_{1,2} J_{-1 / 3}\left(\xi_{2}\right)+\gamma_{2,2} J_{1 / 3}\left(\xi_{2}\right)\right\}+\frac{B(s, n)}{n} .
$$

For $\alpha<s<\beta$, and $\left|\xi_{j}(s)\right|>N, j=1,2$,

$$
y_{2}\left(s, \lambda_{n}\right)\left\{\begin{array}{l}
=\Psi_{2}(s) \xi_{2}^{-1 / 6}(s)\left\{e^{i \xi_{2}(s)}[1]+e^{-i \xi_{2}(s)}[i]\right\}, \\
=(-1)^{n} \Psi_{1}(s) \xi_{1}^{-1 / 6}(s)\left\{e^{i \xi_{1}(s)}[1]+e^{-i \xi_{1}(s)}[i]\right\} .
\end{array}\right.
$$

For $\left|\xi_{1}(s)\right| \leqq N$,

$$
y_{2}\left(s, \lambda_{n}\right)=(-1)^{n} \Psi_{1}(s) \xi_{1}^{1 / 3}(s)\left\{\gamma_{1,2} J_{-1 / 3}\left(\xi_{1}\right)+\gamma_{2,2} J_{1 / 3}\left(\xi_{1}\right)\right\}+\frac{B(s, n)}{n} .
$$

For $\beta<s \leqq b$, and $\left|\xi_{1}(s)\right|>N$,

$$
y_{2}\left(s, \lambda_{n}\right)=(-1)^{n} \Psi_{1}(s) \xi_{1}^{-1 / 6}(s) e^{i \xi_{1}(s)}[1] .
$$

The significance of the symbols is the same as in Theorem 1.

14. The forms of $u_{2}(x)$ and $u_{-2}(x)$ when $\lambda$ is bounded from the characteristic values. The formula for the coefficient $c_{1,2}$ as it is given in (7.3), is, to the extent of its explicitness, indistinguishable from the left-hand member of the characteristic Equation (13.2). Zeros of this coefficient therefore also exist, and are distributed in accordance with a formula like (13.3). Stated otherwise, they are asymptotically represented by the characteristic values. This permits the following observations: If, with the choice of an arbitrarily small positive constant $\rho$ the circles with radius $\rho / 2$ and centers at the points $\lambda_{n}$ are drawn, the zeros of $c_{1,2}$ which are of sufficiently large modulus lie 
within these circles. Therefore if $\lambda$ is constrained from lying within any circle with the radius $\rho$ and a center $\lambda_{n}$ it will be uniformly bounded from the zeros in question. For such $\lambda$ it is known, [5] that the ratio of $c_{1,2}$ to either one of the exponentials that appears in its formula (7.3) is bounded from zero.

We shall suppose, in the following discussion, that $\lambda$ is constrained in the manner described. The coefficients $\beta_{1,2}$ and $\beta_{2,2}$ as well as $c_{1,2}$ are then of the order of $e^{ \pm \pi \lambda / 2}$, as may be seen from the relations (7.3) and (7.4). In accordance with this and the formula $(7.5 \mathrm{~d})$ we have $v_{2}(x)=e^{ \pm \pi \lambda / 2} B(x, \lambda)$ when $x$ is on the interval $\left(x_{0}, x_{1}\right)$ whereas by (7.5e) the corresponding evaluation when $x>x_{1}$ is $v_{2}(x)=e^{ \pm \pi \lambda / 2-i \xi(x)} B(x, \lambda)$. We are prepared now to determine the forms of $u_{2}(x)$ on the sub-intervals that were not considered in $\$ 12$.

When $x$ is on the sub-interval $\left(x_{0}, x_{1}\right)$ the Equation (12.1) may be put into the form

$$
U_{2,4}(x)=F_{2,4}(x, \lambda)+\frac{1}{\lambda} \int_{x_{0}}^{x} Q(x, t, \lambda) U_{2,4}(t) d t,
$$

with

$$
\begin{aligned}
& U_{2,4}(x)=e^{\mp \pi \lambda / 2} u_{2}(x), \\
& V_{2,4}(x)=e^{\mp \pi \lambda / 2} v_{2}(x),
\end{aligned}
$$

and

$$
F_{2,4}(x, \lambda)=V_{2,4}(x)+\frac{1}{\lambda} \int_{-x_{a}}^{x_{0}} Q(x, t, \lambda) U_{2,4}(t) d t .
$$

The table $1_{4}$ of $\S 10$ with $U(t)$ taken to be $U_{2,4}(t)$ is applicable, along with the table $2{ }_{4}$ below.

\begin{tabular}{c|c|}
\hline$t$-interval & $\left(-x_{a},-x_{1}\right)$ \\
\hline$Q(x, t, \lambda)$ & $\lambda^{1 / 6} \phi^{-1 / 2}(t) e^{ \pm \pi \lambda / 2-\pi \lambda / 2+i \xi(t)} B(x, t, \lambda)$ \\
\hline$U_{2,4}(t)$ & $\lambda^{-1 / 6} \phi^{-1 / 2}(t) e^{\mp \pi \lambda / 2+\pi \lambda / 2-i \xi(t)} B(t, \lambda)$ \\
\hline
\end{tabular}

\section{TABLE 24}

These entries show that the conditions (8.6a) and (8.6b) are fulfilled, and hence that

$$
u_{2}(x)=v_{2}(x)+\frac{e^{ \pm \pi \lambda / 2} B(x, \lambda)}{\lambda}, \quad \text { for } x_{0} \leqq x \leqq x_{1} .
$$

When $x$ is on the fifth sub-interval $\left(x_{1}, x_{b}\right)$ the abbreviations 


$$
\begin{aligned}
& U_{2,5}(x)=\lambda^{1 / 6} \phi^{1 / 2}(x) e^{\mp \pi \lambda / 2+i \xi(x)} u_{2}(x), \\
& V_{2,5}(x)=\lambda^{1 / 6} \phi^{1 / 2}(x) e^{\mp \pi \lambda / 2+i \xi(x)} v_{2}(x),
\end{aligned}
$$

serve to give the Equation (12.1) the form

$$
U_{2,5}(x)=F_{2,5}(x, \lambda)+\frac{1}{\lambda} \int_{x_{1}}^{x} K_{1}(x, t, \lambda) U_{2,5}(t) d t,
$$

with

$$
F_{2,5}(x, \lambda)=V_{2,5}(x)+\frac{1}{\lambda} \int_{-x_{a}}^{x_{1}} K_{1}(x, t, \lambda) U_{2,5}(t) d t .
$$

The Table $1_{5}$ with $U(t)$ taken to be $U_{2,5}(t)$ along with the Table $2_{5}$ below, are applicable.

\begin{tabular}{c|c}
$t$-interval & $\left(-x_{a},-x_{1}\right)$ \\
\hline$Q(x, t, \lambda)$ & $\phi^{-1 / 2}(x) \phi^{-1 / 2}(t) e^{ \pm \pi \lambda / 2-\pi \lambda / 2-i \xi(x)+i \xi(t)} B(x, t, \lambda)$ \\
\hline$U_{2,5}(t)$ & $e^{\mp \pi \lambda / 2+\pi \lambda / 2} B(t, \lambda)$ \\
\hline
\end{tabular}

TABLE 25

One finds that

$$
u_{2}(x)=v_{2}(x)+\frac{e^{ \pm \pi \lambda / 2-i \xi(x)} B(x, \lambda)}{\lambda^{7 / 6} \phi^{1 / 2}(x)}, \quad \text { for } x_{1}<x \leqq x_{b} .
$$

The results (14.2) and (14.3) make the formulas (7.5d) and (7.5e) for $v_{2}(x)$ again applicable to $u_{2}(x)$, but they do that only because the coefficients $\beta_{1,2}, \beta_{2,2}$ and $c_{1,2}$ are not small relative to the factor $e^{ \pm \pi \lambda / 2}$. That, it will be recalled, was assured by the bounding of $\lambda$ from the characteristic values.

The deduction of the forms of $u_{-2}(x)$ on the intervals $\left(-x_{1},-x_{0}\right)$ and $\left(-x_{a},-x_{1}\right)$ can be made in an analogous way. The remarks of $\S 13$ relative to this are again applicable. The forms of $u_{-2}(x)$ in the stated intervals are found to be those given for $v_{-2}(x)$ in (7.5b) and (7.5a).

A restatement of the results that have thus been obtained for the normalized Equation (4.2), to make them applicable to the differential equation in its originally given form (1.1), is accomplished, as in $\$ 11$, by the use of the relations (11.2), (11.3) and (11.4). The conclusion to which this leads is the following one:

THEOREm 3. The differential Equation (1.1), has, when $0 \leqq \arg \lambda<\pi$ and $|\lambda|$ is sufficiently large, a solution $y_{2}(s)$ which is represented when $a \leqq s<\beta$ and 
$\left|\xi_{1}(s)\right|>N$, and a solution $y_{-2}(s)$ which is represented when $\alpha<s \leqq b$ and $\left|\xi_{2}(s)\right|>N$, in the manner

$$
y_{ \pm 2}(s) \sim\left\{\frac{x^{2}(s)-1}{\theta^{2}(s)}\right\}^{1 / 4} w_{ \pm 2}\left((i \lambda)^{1 / 2} x(s), \frac{i \lambda-1}{2}\right),
$$

where $w_{2}(z, \kappa)$ and $w_{-2}(z, \kappa)$ are certain Weber functions. When $\lambda$ is furthermore uniformly bounded from the characteristic values these representations are valid over the entire interval $a \leqq s \leqq b$.

In consequence of this the forms of these solutions in the several parts of the intervals in question are the following ones:

For $a \leqq s<\alpha$ and $\left|\xi_{2}(s)\right|>N$;

$$
\begin{aligned}
y_{2}(s) & =\Psi_{2}(s) \xi_{2}^{-1 / 6}(s) e^{i \xi_{2}(s)}[1] \\
y_{-2}(s) & =\Psi_{2}(s) \xi_{2}^{-1 / 6}(s)\left\{c_{1,2}[1] e^{-i \xi_{2}(s)}+\left(c_{1,2}[\delta]+c_{2,2}[1]\right) e^{i \xi_{2}(s)}\right\} .
\end{aligned}
$$

For $\left|\xi_{2}(s)\right| \leqq N$;

$$
\begin{gathered}
y_{2}(s)=\Psi_{2}(s) \xi_{2}^{1 / 3}(s)\left\{\gamma_{1,2} J_{-1 / 3}\left(\xi_{2}\right)+\gamma_{2,2} J_{1 / 3}\left(\xi_{2}\right)\right\}+\frac{B(s, \lambda)}{\lambda} \\
y_{-2}(s)=\Psi_{2}(s) \xi_{2}^{1 / 3}(s)\left\{\beta_{1,2} J_{-1 / 3}\left(\xi_{2}\right)+\beta_{2,2} J_{1 / 3}\left(\xi_{2}\right)\right\}+\frac{c_{1,2} B(s, \lambda)}{\lambda} .
\end{gathered}
$$

For $\alpha<s<\beta$, and $\left|\xi_{j}(s)\right|>N, j=1,2$;

$$
y_{2}(s)=\Psi_{l}(s) \xi_{l}^{-1 / 6}(s)\left\{e^{i \xi_{2}(s)}[1]+e^{-i \xi_{2}(s)}[i]\right\},
$$

$$
y_{-2}(s)=\Psi_{l}(s) \xi_{l}^{-1 / 8}(s)\left\{e^{i \xi_{1}(s)}[1]+e^{-i \xi_{1}(s)}[i]\right\}, \quad \text { with } l \text { either } 1 \text { or } 2 .
$$

For $\left|\xi_{1}(s)\right| \leqq N$;

$$
y_{2}(s)=\Psi_{1}(s) \xi_{1}^{1 / 3}(s)\left\{\beta_{1,2} J_{-1 / 3}\left(\xi_{1}\right)+\beta_{2,2} J_{1 / 3}\left(\xi_{1}\right)\right\}+\frac{c_{1,2} B(s, \lambda)}{\lambda},
$$

$$
y_{-2}(s)=\Psi_{1}(s) \xi_{1}^{1 / 3}(s)\left\{\gamma_{1,2} J_{-1 / 3}\left(\xi_{1}\right)+\gamma_{2,2} J_{1 / 3}\left(\xi_{1}\right)\right\}+\frac{B(s, \lambda)}{\lambda} .
$$

For $\beta<s \leqq b$, and $\left|\xi_{1}(s)\right|>N$;

$$
\begin{aligned}
y_{2}(s) & =\Psi_{1}(s) \xi_{1}^{-1 / 6}(s)\left\{\left(c_{1,2}[\delta]+c_{2,2}[1]\right) e^{i \xi_{1}(s)}+c_{1,2}[1] e^{-i \xi_{1}(s)}\right\} \\
y_{-2}(s) & =\Psi_{1}(s) \xi_{1}^{-1 / 6}(s) e^{i \xi_{1}(s)}[1] .
\end{aligned}
$$

The coefficients $c_{1,2}, c_{2,2}, \beta_{1,2}$ and $\beta_{2,2}$ are given by the Formulas (7.3) and (7.4). Other notation, and the procedure for obtaining the forms of the derivatives are as was described in Theorem 1. 


\section{REFERENCES}

1. Nathan Schwid, The asymptotic forms of the Hermite and Weber functions, Trans. Amer. Math. Soc. vol. 37 (1935) pp. 339-362.

2. A. Erdélyi, M. Kennedy, and J. L. McGregor, Parabolic cylinder functions of large order, Journal of Rational Mechanics and Analysis vol. 3 (1954) pp. 459-485.

3. W. E. Johnson, Asymptotic solutions of a linear second order differential equation with two turning points, Dissertation, University of Wisconsin, 1952; Bull. Amer. Math. Abstract 58-6-633.

4. R. E. Langer, On the asymptotic solutions of differential equations, with an application to the Bessel functions of large complex order, Trans. Amer. Math. Soc. vol. 34 (1932) pp. 447-480.

5. - On the zeros of exponential sums and integrals, Bull. Amer. Math. Soc. vol. 37 (1931) pp. 213-239.

Mathematics Research Center, U. S. Army, University of Wisconsin, Madison, Wis. 